and medium-distance migrants will be selected to migrate less far. After the recent mild winters in Europe there have been many reports from central Europe of short-distance migrants wintering in their breeding areas. The success of the late-arriving, long-distance migrants depends on the degree of competition from resident birds and from short-distance migrants that have already established territories. Increases in the number of resident birds are likely to be deleterious for long-distance migrants. With further man-induced environmental changes on the cards, the future of many species could well depend on their ability to adapt in the way the blackcap seems to have done.

William J. Sutherland is at the School of Biological Sciences, University of East Anglia, Norwich NR4 7TJ, UK.

\title{
CHEMISTRY
}

\section{Vocabulary for fuzzy symmetry}

\section{Patrick W. Fowler}

SYMMETRY arguments are standard tools of the trade in theoretical chemistry and physics. Their power lies in the way they deliver reassuringly definite predictions. Sulphur hexafluoride $\left(\mathrm{SF}_{6}\right)$ is centrosymmetric and octahedral at equilibrium. Can it support a permanent dipole moment? (No.) Are all six fluorine nuclei magnetically equivalent? (Yes.) Is the molecule optically active? (No.) Should its breathing vibration give rise to a peak in the Raman spectrum? (Yes.) Group theory ${ }^{1.2}$ has all the answers. However, the world is rarely so simple, and Zabrodsky et al. have developed a system of "continuous symmetry measures"3 to deal with the murky area of nearly symmetrical or slightly asymmetrical objects. They argue that we should add words like perhaps, maybe and sometimes to the yes-or-no, now-or-never vocabulary of point-group symmetry.

The new approach can be illustrated with the simplest plane figure, the triangle. A rigid, equilateral triangle lying in the horizontal plane has various elements of symmetry. It can be brought into a physically indistinguishable configuration (one in which no interparticle distance is altered) by rotations of $120^{\circ}$ clockwise or anticlockwise about a vertical axis, $180^{\circ}$ about one of three horizontal axes, reflection in the horizontal plane, or reflection in one of three vertical planes. Isosceles triangles have only two reflection planes and one twofold axis; scalene triangles have only the horizontal reflection plane. In the notation used by chemists, the three objects belong to the three distinct point groups $D_{3 h}, C_{2 v}$ and $C_{s}$, respectively.

The question addressed by Zabrodsky et al. can be put this way: what happens to the symmetry description if we start with an equilateral triangle and gradually distort it? Nudge one of the vertices along a radius and the triangle is now isosceles. Nudge the vertex along a tangent and the triangle becomes scalene. For a traditionalist, all three cases are quite different and are described by

distinct groups, because a symmetry element is lost as soon as the vertex starts to move.

In the continuous approach, the three triangles are described by a gradual movement away from symmetry elements, and each instantaneous con-

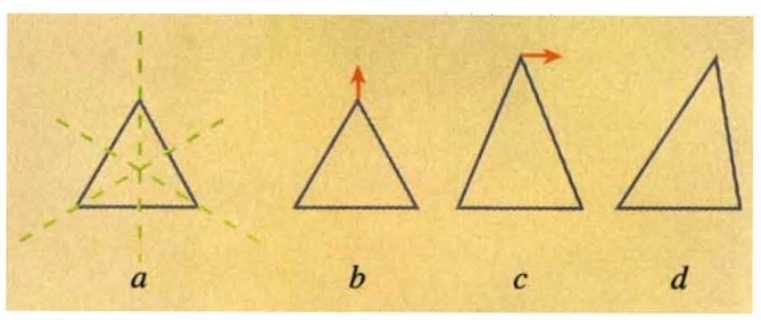

An equilateral triangle (a) has four axes and four planes of symmetry. Small displacements $(b, c, d)$ have a catastrophic effect on the name we attach to the triangle and its symmetry group, but may hardly affect the properties.

figuration is characterized by numerical measures $S\left(C_{3}\right), S\left(C_{2}\right), S(\sigma)$ of the deviation from a rotational or mirror symmetry. The procedure for assigning these indices is straightforward, depending on a weighted sum of squares of distances between points and their images in a hypothetical symmetrized set representing the nearest object with the desired symmetry. Things get slightly more complicated in three dimensions, because it is not obvious where the missing axes or planes should be, but a preliminary application to three dimensions is given in the paper. A remaining technical issue is whether the continuous symmetry measure (CSM) can be extended to describe deviation from a point group rather than from a single element.

Why are these apparently trivial geometrical manipulations significant for chemistry and molecular physics? Mainly because chemists still use a largely mechanical model of the molecule. The story is that electrons are light and nuclei are heavy. The nuclear framework vibrates, rotates and translates, held together by electronic glue and electronic springs, and where the nuclei go the electrons are sure to follow. At 4QD, UK. each nuclear geometry the electron distribution can be found by solving the Schrödinger equation with clamped nuclei. Breakdown of this simple BornOppenheimer picture can be important in certain situations, but the approximation does describe most molecules in their ground electronic states.

A vibrating nonlinear molecule is continually sampling geometries of less than the nominal point group symmetry, and one use of CSM indices will be in parametrizing the property surfaces that describe the new electronic properties that arise in the anisotropic nonequilibrium geometries. An example would be the dipole moment of $\mathrm{SF}_{6}$ induced by stretching one bond. CSM can also represent the small changes in equilibrium geometry caused by chemical substitution, for example, the (slight) loss of sixfold rotational symmetry in the benzene ring of toluene ${ }^{3}$. This can already be done using internal displacement coordinates based on a single highsymmetry structure, but CSM seems to offer an unbiased way of representing simultaneously the relationship between a given structure and a whole set of conceivable symmetric forms. It will be interesting to see if its proponents can link the CSM concept to the group theory of floppy molecules $^{2}$, and to the study of chirality measures ${ }^{4}$.

One case that the new scheme does not seem to cover is where the symmetry of the electron distribution is lower than that of the nuclear framework. For example, when a rigid molecule is polarized by a uniform field the electron cloud can be strongly distorted, but the nuclei may hardly shift. The molecule has certainly lost symmetry in this process, but in a way that is invisible to CSM.

The problem of any symmetry-based approach is that a broken symmetry implies the existence of a property, but cannot predict its value. Continuous symmetry is a partial answer to this problem, in that a small CSM index implies a 'small' value of the property, but with a constant of proportionality that remains to be established by calculation or experiment in individual cases. $\square$

Patrick Fowler is in the Department of Chemistry, University of Exeter, Exeter EX4

1. Cotton, F. A. Chemical Applications of Group Theory 3rd edn (Wiley, New York, 1990)

2. Bunker. P. R. Molecular Symmetry and Spectroscopy (Academic, Orlando, 1979).

3. Zabrodsky, H., Peleg, S. \& Avnir, D. J. Am. chem. Soc 114, 7843-7851 (1992)

4. Buda, A. B., Auf der Deyde, T. \& Mislow, K. Angew. Chemie Int. Ed. Engl. 31, 989-1007 (1992).

NATURE · VOL 360 - 17 DECEMBER 1992 